\title{
High titer of antibody against the SARS-CoV-2 spike protein among patients receiving neutralizing antibody cocktail therapy with REGN-COV
}

\author{
Hiroaki Sasaki $^{1}$ (1) $\cdot$ Nobuyuki Miyata $^{1} \cdot$ Yukihiro Yoshimura $^{1} \cdot$ Natsuo Tachikawa $^{1}$
}

Received: 24 December 2021 / Accepted: 8 February 2022 / Published online: 18 February 2022

(c) The Author(s), under exclusive licence to Springer-Verlag GmbH Germany 2022

\begin{abstract}
Purpose Casirivimab/imdevimab (REGN-COV), a cocktail of neutralizing antibodies against the receptor-binding domain of the severe acute respiratory syndrome coronavirus 2 (SARS-CoV-2) spike protein, was shown to be an effective treatment and post-exposure prophylaxis measure for coronavirus disease 2019 (COVID-19). We assessed the antibody titers among patients who received REGN-COV with the purpose of evaluating this therapeutic and prophylactic option from the serological point of view.

Methods We collected serological data of patients with COVID-19 who were treated with REGN-COV $1200 \mathrm{mg}$ (casirivimab $600 \mathrm{mg} / \mathrm{imdevimab} 600 \mathrm{mg}$ ). Antibody titers were assessed within $24 \mathrm{~h}$ before and within $48 \mathrm{~h}$ after the administration of REGN-COV using ARCHITECT SARS-CoV-2 immunoglobulin $(\mathrm{Ig}) \mathrm{G}\left(\mathrm{IgG}_{\mathrm{NC}}\right)$, which is against nucleocapsid protein, and ARCHITECT SARS-CoV-2 IgG II Quant ( $\left.\operatorname{IgG}_{\mathrm{SP}}\right)$, which is against spike protein.

Results A total of nine patients were evaluated. $\mathrm{IgG}_{\mathrm{SP}}$ was elevated after REGN-COV administration with a median of 208,370 Arbitrary Units/mL while simultaneous $\operatorname{IgG}_{\mathrm{NC}}$ remained low. With the simple linear regression model, the $\mathrm{IgG}_{\mathrm{SP}}$ after the REGN-COV administration was correlated with the reciprocal of ideal body weight.

Conclusion The high titer of $\mathrm{IgG}_{\mathrm{SP}}$ supports the clinical benefit of therapeutic and prophylactic use of REGN-COV from the serological point of view.
\end{abstract}

Keywords COVID-19 $\cdot$ SARS-CoV-2 $\cdot$ REGN-COV $\cdot$ Casirivimab/imdevimab $\cdot$ Antibody titer

The pandemic of novel coronavirus disease 2019 (COVID$19)$, caused by the severe acute respiratory syndrome coronavirus 2 (SARS-CoV-2), is an ongoing threat globally. With the hypothesis that complications and death from COVID-19 emanate from the SARS-CoV-2 viral burden and that reducing this burden leads to clinical benefit, a clinical trial of casirivimab/imdevimab (REGN-COV), a cocktail of neutralizing antibodies targeting the receptor-binding domain (RBD) of the SARS-CoV-2 spike protein, was performed, which showed therapeutic benefit in reducing viral load and lowering the risk of hospitalization or death from COVID19 [1]. Subcutaneous REGEN-COV was also reported to

Hiroaki Sasaki

sasaki.hiro.21@gmail.com

1 Department of Infectious Diseases, Yokohama Municipal Citizen's Hospital, 1-1, Mitsuzawanishimachi, Yokohama City, Kanagawa, Japan prevent symptomatic COVID-19 and asymptomatic SARSCoV-2 infection in previously uninfected household contacts [2]. There are several serological assays for COVID-19, and they have been increasingly used to aid the diagnosis of SARS-CoV-2 infection and to evaluate the antibody response in individuals that have received the COVID-19 vaccine [3, 4]. We believe that to discuss the neutralizing antibody cocktail in comparison with other therapeutic and prophylactic options, common laboratory quantitative assays, such as antibody titer, are helpful. To our knowledge, however, there have been no reports of serological assessment of antibody cocktails among patients with COVID-19 in clinical settings. Since we have assessed patients' immunological status by measuring antibody titers during the treatment of patients with COVID-19, we have accumulated data regarding antibody titers among patients with COVID19 , including those who were treated with REGN-COV. Herein, we present those data for a better understanding of this promising therapeutic and prophylactic option. 
This study was approved by the ethics committee of Yokohama Municipal Citizen's Hospital (no. 21-12-01) and conducted in accordance the ethical standards laid down in the 1964 Declaration of Helsinki and its later amendments. Informed consent was waived due to the retrospective nature of this study and ethical consideration for the use of clinical information was made based on the institutional opt-out policy (https://yokohama-shiminhosp.jp/introduction/iryou kenkyu.html). We retrospectively collected the data of patients with COVID-19 who were admitted to Yokohama Municipal Citizen's Hospital and were treated with REGN-COV. The dosing of REGN-COV was $1200 \mathrm{mg}$ (casirivimab $600 \mathrm{mg}$ / imdevimab $600 \mathrm{mg}$ ) in accordance with authorization by the Ministry of Health, Labour and Welfare of Japan. During hospitalization, we assessed patients' immunological status using ARCHITECT SARS-CoV-2 IgG and ARCHITECT SARSCoV-2 IgG II Quant (Abbott, IL, USA) with cutoff points of 1.4 sample result divided by the calibrator result $(\mathrm{S} / \mathrm{C})$ and 50 Arbitrary Units (AU)/mL, respectively, according to the recommendation by the manufacturer. The former kit identifies IgG against nucleocapsid ( $\operatorname{IgG}_{\mathrm{NC}}$ ), which was expected to be unaffected by REGN-COV administration and reflect the immune response to viral infection. The latter kit identifies IgG against the RBD in the spike protein of SARS-CoV-2 $\left(\mathrm{IgG}_{\mathrm{SP}}\right)$, which was expected to be affected with REGN-COV administration. We collected both antibody titers within $24 \mathrm{~h}$ before (pre-) and within $48 \mathrm{~h}$ after (post-) the administration of REGN-COV. With an assumption that post-IgG $\mathrm{SP}_{\mathrm{SP}}$ titers should be related to body mass with the same dose, we adopted the simple linear regression analysis to evaluate the correlation of post- $\mathrm{IgG}_{\mathrm{SP}}$ with the reciprocal of real and ideal body weight (IBW). Statistical analysis was done using SAS Institute JMP version 15.2 .1 and $p$ value $<0.05$ was considered as statistically significant.

In total, post- $\operatorname{IgG}_{\mathrm{SP}}$ titers were available in nine patients, which were included in our observation (Table 1). The titers of pre- and post- $\operatorname{IgG}_{\mathrm{NC}}$ were low in all patients. The titers of pre- $\operatorname{IgG}_{\mathrm{SP}}$ were negative in seven patients. Two patients had positive pre- $\mathrm{IgG}_{\mathrm{SP}}$. Both of them, whose pre- $\mathrm{IgG}_{\mathrm{SP}}$ titers were 231.2 AU/mL and 699.9 AU/mL, had already received two doses of the SARS-COV-2 vaccine (BNT162b2) with the first dose 3 months and 5 months prior to the admission, respectively. The post-IgG $\mathrm{SP}_{\mathrm{SP}}$ titers were positive in all patients. The exact titers level was unavailable in one patient, although it was confirmed to be more than $80,000 \mathrm{AU} / \mathrm{mL}$. As for the other eight patients, the titers were ranging from $128,436 \mathrm{AU} / \mathrm{mL}$ to $332,710 \mathrm{AU} / \mathrm{mL}$ with a median of $208,370 \mathrm{AU} / \mathrm{mL}$. With the simple linear regression analysis, post-IgG $\mathrm{ISP}_{\mathrm{SP}}$ titers were correlated with the reciprocal of IBW with the predictive equation as follows (Fig. 1; $p=0.006, R^{2}=0.743$ ):

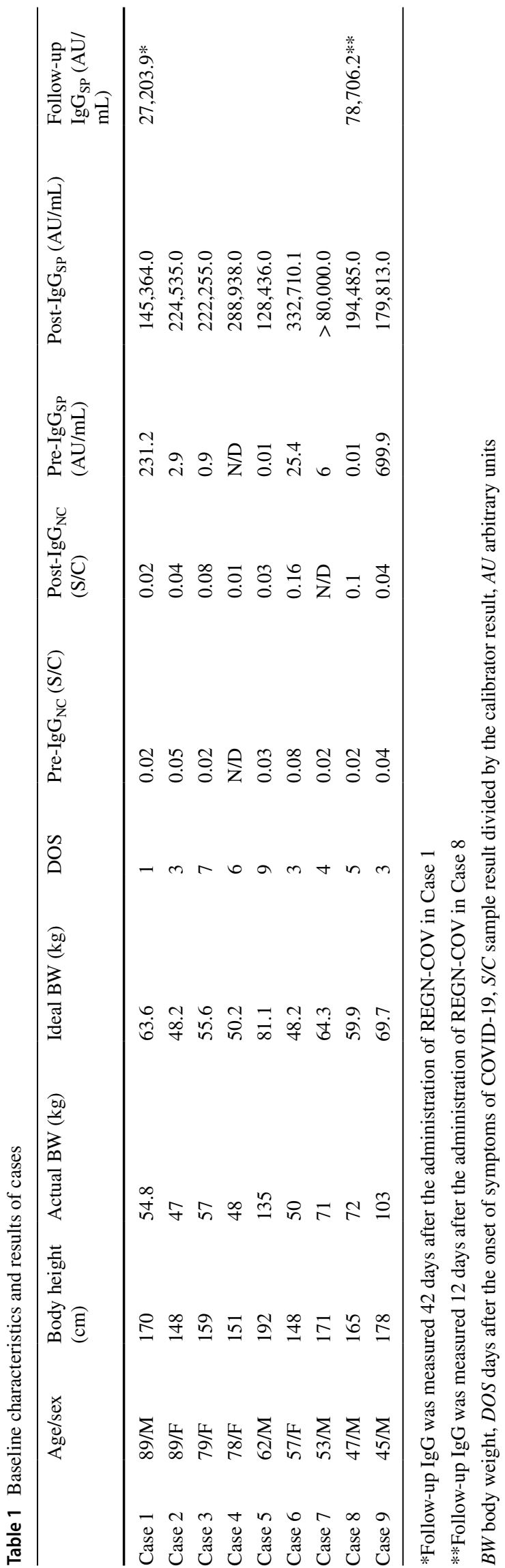




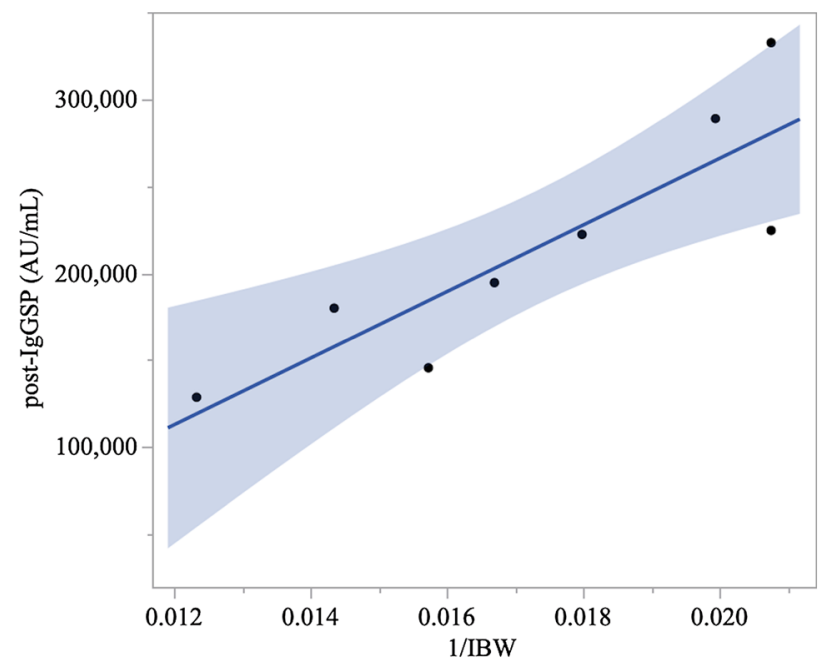

Fig. 1 Distribution of the reciprocal of ideal body weight and post$\mathrm{IgG}_{\mathrm{SP}}$ titers. The univariate linear regression model showed that post$\mathrm{IgG}_{\mathrm{SP}}$ titer was significantly correlated with the reciprocal of ideal body weight $\left(p=0.012, R^{2}=0.752\right)$ with the prediction equation: $\mathrm{IgG}_{\mathrm{SP}}(\mathrm{AU} / \mathrm{mL})=2.05 \times 10^{7} \times 1 /[$ ideal body weight $(\mathrm{kg})]-144,120$. The blue area indicates $95 \%$ confidence interval

Estimated Post $-\mathrm{IgG}_{\mathrm{SP}}(\mathrm{AU} / \mathrm{mL})$

$$
=1.92 \times 10^{7} \times 1 / \mathrm{IBW}(\mathrm{kg})-117,669
$$

Post- $\mathrm{IgG} \mathrm{G}_{\mathrm{SP}}$ titers were not associated with the reciprocal of actual body weight $(p=0.080)$. In one patient (Case 8), $\mathrm{IgG}_{\mathrm{SP}}$ titers were followed 12 days after the REGN-COV administrations and showed a $60 \%$ decrease (from 194,485 $\mathrm{AU} / \mathrm{mL}$ to $78,706.2 \mathrm{AU} / \mathrm{mL}$ ). In another patient (Case 1), $\mathrm{IgG}_{\mathrm{SP}}$ titers were followed 42 days after the REGNCOV administrations and showed an $81 \%$ decrease (from $145,364 \mathrm{AU} / \mathrm{mL}$ to $27,203.9 \mathrm{AU} / \mathrm{mL}$ ). No patients experienced adverse events related to REGN-COV infusion. No patients developed a severe condition and all patients recovered and were discharged from the hospital.

In our observation, the post-IgG $\mathrm{S}_{\mathrm{SP}}$ titer was remarkably high. This titer could be regarded as that of passive immunity with REGN-COV administration rather than the patients' immune reaction, because $\mathrm{IgG}_{\mathrm{NC}}$ titer, which starts to increase almost simultaneously with $\operatorname{IgG}_{\mathrm{SP}}$ titer [5], was not elevated at the time of post-IgG $\mathrm{IPP}_{\mathrm{SP}}$ titer assessment. The post- $\operatorname{IgG}_{\mathrm{SP}}$ titer appeared to be higher than those in infected patients and vaccinated individuals based on the previous reports. For example, in the longitudinal assessment of the titer of $\operatorname{IgG}_{\mathrm{SP}}$ among COVID-19 inpatients, the mean titer increased to $59,396 \mathrm{AU} / \mathrm{mL}$ with a decline after 27-days post-COVID-19 reverse transcription-polymerase chain reaction (RT-PCR) testing [3]. In our previous report on the $\mathrm{IgG}_{\mathrm{SP}}$ titer response after the
COVID-19 vaccine (BNT162b2) among healthcare workers, the median titer of $\mathrm{IgG}_{\mathrm{SP}}$ was $23,489 \mathrm{AU} / \mathrm{mL} 35$ days after the first dose of the vaccine [4].

Since correlation of anti-spike protein IgG and neutralizing antibody responses have already been shown [6-8], high titer of $\mathrm{IgG}_{\mathrm{SP}}$ among REGN-COV administered patients reflect their potent neutralizing capability, which, we believe, have clinical significance. The fact that the titer was higher in the patients with cocktail therapy than the infected patients might support the clinical efficacy of REGN-COV in contrast to convalescent plasma. In a randomized, multicenter single-blind trial, which enrolled a total of 511 patients, the administration of COVID-19 convalescent plasma with "a high titer of antibody against SARS-CoV-2" to high-risk outpatients within 1 week after the onset of symptoms of COVID-19 failed to prevent disease progression [9]. Although there are no data concerning the antibody titer level after administration of convalescent plasma, it was expected to be much lower than those with REGN-COV administration, since only one unit of plasma was administered and convalescent plasma itself likely had a lower titer in the first place.

In phase 3 clinical trial, subcutaneous REGN-COV prevented symptomatic COVID-19 and asymptomatic SARSCoV-2 infection in a post-exposure prophylaxis setting [2]. It is reported that immune protection is highly predictable by the neutralization level [10]. Thus, the fact that the titer of $\mathrm{IgG}_{\mathrm{SP}}$ was higher in the patients with cocktail therapy than the vaccinated individual could reflect the superiority of REGN-COV in prophylaxis not only in rapidity but also strength, especially in unvaccinated patients or individuals with poor humoral response to vaccination, such as the Case 1 in our study.

One of the major limitations of this study is the small number of subjects, resulting in a wide $95 \%$ confidence interval of the predictive equation of the estimated $\operatorname{IgG}_{\mathrm{SP}}$ titer. Thus, the quantitatively of our study should be treated carefully and needs to be revised by analysis of a larger population. Another limitation is the lack of followup data. However, the above-mentioned clinical trial of REGN-COV [1] provided the pharmacokinetics parameters, which was linear and dose-proportional, suggesting the potential of measurement of initial $\mathrm{IgG}_{\mathrm{SP}}$ titer in predicting the duration of passive immunity. To prove this, a follow-up titer of $\mathrm{IgG}_{\mathrm{SP}}$ and clinical data are needed.

\section{Acknowledgements None.}

Author contributions All the authors meet the ICMJE authorship criteria. HS designed the study and wrote the manuscript. NM, NT, and YY commented on the manuscript and approved the final version. 
Funding The authors did not receive support from any organization for the submitted work.

\section{Declarations}

\section{Conflict of interest None.}

Ethics approval This study was approved by the ethics committee of Yokohama Municipal Citizen's Hospital (no. 21-12-01).

Consent to participate Informed consent was waived due to the retrospective nature of this study and ethical consideration for the use of clinical information was made based on the institutional opt-out policy (https://yokohama-shiminhosp.jp/introduction/iryoukenkyu.html).

Consent to publish Informed consent was waived due to the retrospective nature of this study and ethical consideration for the use of clinical information was made based on the institutional opt-out policy (https:// yokohama-shiminhosp.jp/introduction/iryoukenkyu.html).

\section{References}

1. Weinreich DM, Sivapalasingam S, Norton T, Ali S, Gao H, Bhore $\mathrm{R}$, et al. REGN-COV2, a neutralizing antibody cocktail, in outpatients with COVID-19. N Engl J Med. 2021;384:238-51. https:// doi.org/10.1056/NEJMoa2035002.

2. O'Brien MP, Forleo-Neto E, Musser BJ, Isa F, Chan KC, Sarkar $\mathrm{N}$, et al. Subcutaneous REGEN-COV antibody combination to prevent COVID-19. N Engl J Med. 2021;385:1184-95. https:// doi.org/10.1056/NEJMoa2109682.

3. Narasimhan M, Mahimainathan L, Araj E, Clark AE, Markantonis J, Green A, et al. Clinical evaluation of the Abbott Alinity
SARS-CoV-2 Spike-Specific Quantitative IgG and IgM assays among infected, recovered, and vaccinated groups. J Clin Microbiol. 2021;59: e0038821. https://doi.org/10.1128/JCM.00388-21.

4. Yoshimura Y, Sasaki H, Miyata N, Miyazaki K, Tachikawa N. Antibody response after COVID-19 vaccine BNT162b2 on health care workers in Japan. J Infect Chemother. 2021;27:1713-5. https://doi.org/10.1016/j.jiac.2021.08.008.

5. Burbelo PD, Riedo FX, Morishima C, Rawlings S, Smith D, Das $\mathrm{S}$, et al. Detection of nucleocapsid antibody to SARS-CoV-2 is more sensitive than antibody to spike protein in COVID-19 patients. medRxiv. 2020. https://doi.org/10.1101/2020.04.20. 20071423.

6. Keech C, Albert G, Cho I, Robertson A, Reed P, Neal S, et al. Phase 1-2 Trial of a SARS-CoV-2 recombinant spike protein nanoparticle vaccine. N Engl J Med. 2020;383:2320-32. https:// doi.org/10.1056/NEJMoa2026920.

7. Poh CM, Carissimo G, Wang B, Amrun SN, Lee CY, Chee RS, et al. Two linear epitopes on the SARS-CoV-2 spike protein that elicit neutralising antibodies in COVID-19 patients. Nat Commun. 2020;11:2806. https://doi.org/10.1038/s41467-020-16638-2.

8. Dolscheid-Pommerich R, Bartok E, Renn M, Kümmerer BM, Schulte B, Schmithausen RM, et al. Correlation between a quantitative anti-SARS-CoV-2 IgG ELISA and neutralization activity. J Med Virol. 2022;94:388-92. https://doi.org/10.1002/jmv.27287.

9. Korley FK, Durkalski-Mauldin V, Yeatts SD, Schulman K, Davenport RD, Dumont LJ, et al. Early convalescent plasma for highrisk outpatients with Covid-19. N Engl J Med. 2021;385:1951-60. https://doi.org/10.1056/NEJMoa2103784.

10. Khoury DS, Cromer D, Reynaldi A, Schlub TE, Wheatley AK, Juno JA, et al. Neutralizing antibody levels are highly predictive of immune protection from symptomatic SARS-CoV-2 infection. Nat Med. 2021;27:1205-11. https://doi.org/10.1038/ s41591-021-01377-8. 